\title{
Mammographie de dépistage et Swiss Medical Board
}

\section{Gianfranco Domenighetti}

Ancien Professeur d'Economie et Politique de la Santé aux Universités de la Suisse Italienne (USI) et de Lausanne (UNIL)

Correspondance: Gianfranco Domenighetti PhD gianfranco.domenighetti[at] usi.ch

\section{Rapport du Swiss Medical Board (SMB), une provocation?}

A partir du début des années 2000 des revues médicales respectées et à audience internationale ont régulièrement publié des études, des méta-analyses et des éditoriaux qui s'interrogeaient sur l'efficacité, les risques et les incertitudes de ce dépistage de masse attribuant notamment la baisse de la mortalité pour cancer du sein principalement aux progrès des traitements plutôt qu'à la détection précoce. Des titres tels que «Il n'est pas faux de dire non» (à l'invitation à participer au dépistage) dans le British Medical Journal, «Repenser la mammographie de dépistage» dans le Journal of the American Medical Association, «Il est temps de renoncer à la mammographie de dépistage?» dans le Canadian Medical Journal, «Screening mammographique, plus d'effets indésirés que de bénéfices» dans le British Medical Journal encore, ont rendu palpable que quelque chose était en train de changer. Le catalyseur du changement a été la prise de conscience que le dépistage a également des risques, et notamment le surdiagnostic (qu'il ne faut pas confondre avec les résultats «faux positifs»), dont l'importance et la prévalence ont été jusque là sousestimées pendant des années. Donc, pour ceux qui suivent régulièrement la presse scientifique, il n'y a 


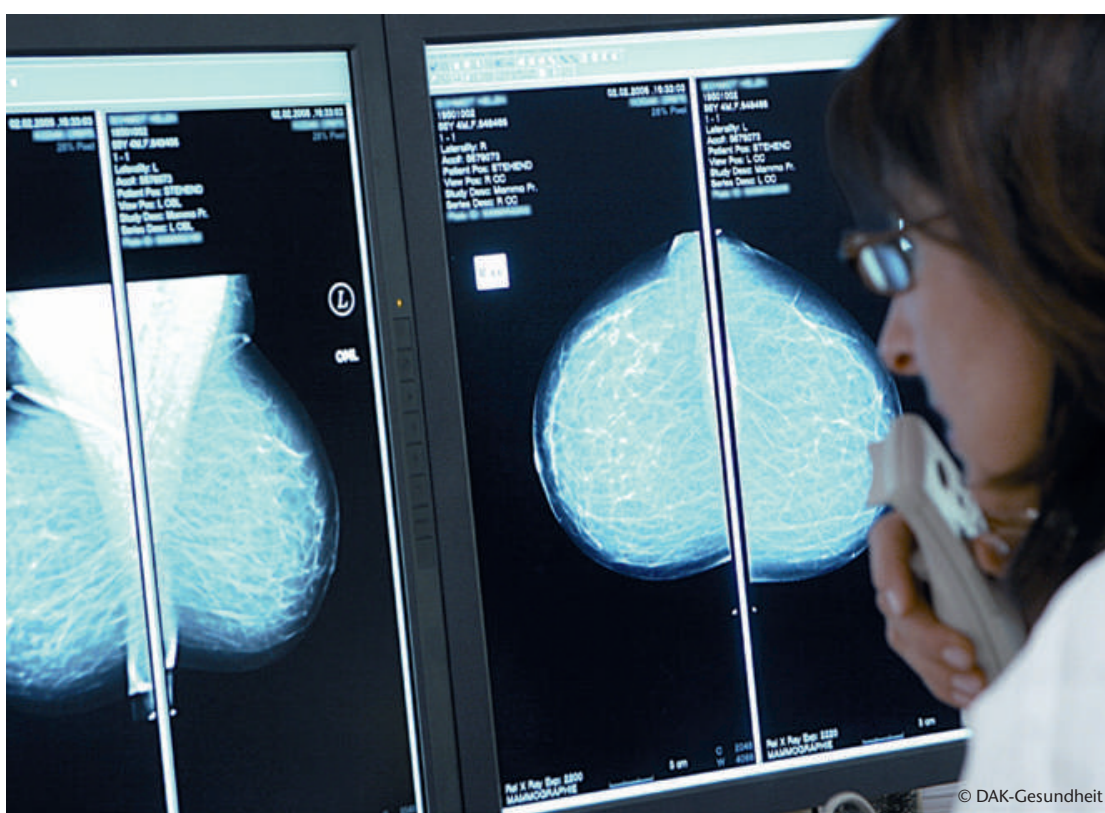

Mammographie de dépistage: un bilan et un choix délicat.

aucun étonnement devant une partie des conclusions du Swiss Medical Board [1], qui remet notamment en question l'efficacité du dépistage systématique du cancer du sein par mammographie chez les femmes subjectivement en bonne santé sans prédisposition familiale. D'ailleurs, juste une semaine après que ce rapport a été rendu public, une étude canadienne sur 90000 femmes suivies pendant 25 ans arrivait à la conclusion que la mortalité par cancer du sein était la même parmi les femmes dépistées et celles qui n'avaient pas participé au dépistage [2].

\section{Mammographie de dépistage: un bilan délicat}

Au delà de ce dernier résultat décevant, le dépistage éloigne le décès par cancer du sein. Selon les études les plus fiables [3-6], sur 1000 femmes de 50 ans qui font régulièrement le dépistage pendant 10-12 ans, entre 0,5 et 2 décès par cancer du sein seront évités, ce qui correspond à une diminution relative de la mortalité de 15 à $25 \%$. Autrement dit pour 998 à 999,5 femmes le dépistage n'aura aucun effet positif sur la mortalité par cancer du sein, mais, au contraire, elles seront soumises aux risques propres à ce type de dépistage c'est-à-dire aux résultats «faux positifs» et au «surdiagnostic». En effet entre 250 et 350 femmes, toujours sur 1000 et après 10-12 ans de dépistage, devront gérer, pendant une semaine voire deux, l'anxiété d'un résultat faux positif et une cinquantaine d'entre elles subiront une biopsie. Bien plus graves seront les conséquences pour les cas de surdiagnostic. On estime que entre 6 et 15 femmes seront diagnostiquées pour des cancers qui n'auraient jamais donné de symptômes et qui n'évolueront pas négativement tout au long de leur vie. Ces femmes seront toutefois traitées inutilement par chirurgie, radiothérapie et chimiothérapie. Le vrai problème est qu' aujourd'hui on n'est pas en mesure de distinguer les tumeurs «surdiagnostiquées» de celles qui nécessitent une prise en charge médicochirurgicale. Donc, si une femme, asymptomatique et sans risque familial, ne veut pas se soumettre au risque d'être «surdiagnostiquée» et traitée inutilement le seul choix possible est celui de ne pas participer au dépistage. Il est important de souligner que le risque de surdiagnostic est bien plus élevé que la chance d'éviter un décès. Si l'on se réfère aux trois études citées auparavant on aura, selon la révision Cochrane 2013 [3], pour un décès évité 10 femmes «surdiagnostiquées» et «surtraitées»; selon l'Independent Review UK 2012 [4], pour un décès évité nous aurons 3 cas de surdiagnostic; selon l'étude Kalager et al. $2010[5,6]$, pour un décès évité 5-15 femmes feront l'objet d'un «surdiagnostic». En général, on estime que le $31 \%$ des cancers du sein dépistés par la mammographie sont des cancers «surdiagnostiqués» qui ne donneront pas de symptômes et n'évolueront pas négativement tout au long de la vie [7]. Il faut aussi noter que plus la technologie de détection devient performante plus il y aura des cas de surdiagnostic.

Pour tous ces motifs, dans la brochure informative sur le dépistage par mammographie du Canton du Tessin [8], on relève que «Les femmes ont des bons〉 motifs pour se soumettre à la mammographie de diagnostic précoce... et des «bons` motifs pour ne pas le faire», car le dépistage a aussi des risques.

\section{Information ou propagande?}

On en arrive au thème crucial de l'information, soulevé aussi par le rapport du SMB. Thomas Perneger a raison: «Laissons les femmes choisir» [9]. Questions: disposent-elles d'une information fondée sur des preuves, qui soit «user-friendly», sans conflits d'intérêts et en mesure de garantir une expression des préférences respectueuse de l'autonomie individuelle? Si on se réfère aux différentes brochures rédigées par ceux qui gèrent directement ou indirectement les dépistages, la réponse est clairement non. La propagande unilatérale en faveur du dépistage [10-12] fait rage depuis une quinzaine d'années, et il n'est pas évident de remettre la montre de l'information à l'heure des preuves après tant d'années de «brainwashing». Et les résultats sont là [13, 14]: plus de $90 \%$ des femmes ne connaissent pas ou surestiment lourdement l'impact de la mammographie sur la diminution de la mortalité par cancer du sein (par exemple en France $45 \%$ des femmes croient que le dépistage va éviter, en 10-12 ans, plus de 100 décès sur 1000 femmes).

Les premières brochures des programmes romands étaient expressément éditées pour induire une augmentation de la participation au dépistage, 
évitant que les femmes puissent se poser beaucoup de questions. En effet, sous le chapitre «Projet information» du Rapport d'activité 2003 de la Fondation Genevoise pour le Dépistage du Cancer du Sein on peut lire à propos de la nouvelle brochure informative: «Le design et le graphisme doivent être rénovés, par rapport à la version actuelle, et le texte plus approprié pour répondre aux attentes élémentaires des femmes, afin de les convaincre à adhérer au programme.» On ajoutait qu'une deuxième brochure «avec une information plus objective [...] sera remise à toutes les personnes qui en feront demande» (!). La brochure actuelle publiée en 2011 pour tous les programmes romands n'est pas un exemple de transparence et de clarté. Elle ne donne, notamment, aucune information quantitative sur les bénéfices et les risques dans une perspective temporelle (on souhaiterait un tableau qui, sur au moins 10 ans de dépistage, compare les bénéfices et les risques en valeurs absolues pour 1000 femmes qui font le dépistage et

\section{Réferences}

1 Swiss Medical Board. Dépistage systématique par mammographie. 15 décembre 2013. www.medicalboard.ch/fileadmin/docs/public/mb/Fachberichte/ 2013-12-15_Rapport_Mammographie_Final_rev.pdf

2 Miller AB, Wall C, Baines CJ, Sun P, To T, Narod SA. Twenty five year follow-up for breast cancer incidence and mortality of the Canadian National Breast Screening Study: randomised screening trial. BMJ. 2014;348:g366.

3 Gøtzsche PC., Jørgensen KJ. Screening for breast cancer with mammography. Cochrane Database of Systematic Reviews. 2013; Issue 6. Art. No.:CD001877. DOI:10.1002/14651858.CD001877.pub5.

4 Independent UK Panel on Breast Cancer Screening. The benefits and harms of breast cancer screening: an independent review. Lancet. 2012;380(9855):1778-86.

5 Kalager M, Zelen M, Langmark F, Adami HO. Effect of screening mammography on breast-cancer mortality in Norway. N Engl J Med. 2010;363(13):1203-10.

6 Welch HG. Screening mammography--a long run for a short slide? N Engl J Med. 2010;363(13):1276-8.

\section{«9,5\% seulement des participantes à un dépistage oncologique ont été informées par leur médecin du risque de surdiagnostic.»}

1000 femmes qui ne le font pas). La réduction de la mortalité est toujours exprimée en termes relatifs ( $25 \%$ de quoi?) et non pas en valeurs absolues. En plus la brochure donne une définition absolument «maison» et pratiquement incompréhensible du surdiagnostic, sans fournir aucune estimation quantitative du risque ni en termes relatifs ni absolus de cet événement indésirable majeur.

S'informer alors chez son médecin? Pas sûr qu'il s'agisse du meilleur choix. Une étude récente a montré que 9,5\% seulement des participantes à un dépistage oncologique ont été informées par leur médecin du risque de surdiagnostic [15]. D'ailleurs la majorité de ces derniers interprètent faussement les statistiques relatives aux dépistages [16].

Je laisse la conclusion au Docteur Maureen Roberts qui a mis en œuvre et dirigé le programme de dépistage d'Edinbourg et qui est malheureusement décédée suite à un cancer du sein alors qu'elle était encore en pleine activité. Il y a 25 ans, quelques jours avant de mourir en juin 1989, elle a écrit un article pour le British Medical Journal [17] dont le titre était «Breast screening: time for a rethink?» Elle soulignait notamment que «The decision must be theirs, and a truthful account of the facts must be made available to the public and the individual patient.».
7 Bleyer A, Welch HG. Effect of three decades of screening mammography on breast-cancer incidence. N Engl J Med. 2012;367(21):19982005.

8 Version en français de la brochure du Canton du Tessin www4.ti.ch/fileadmin/DSS/DSP/UPVS/PDF/Progetti/ MammografiaFRA_2010.pdf

9 Perneger T. Mammographie de dépistage: laissons les femmes choisir. Bull Méd Suisses. 2014;95(6):197.

10 Gøtzsche PC, Hartling OJ, Nielsen M, Brodersen J, Jørgensen KJ. Breast screening: the facts - or maybe not. BMJ. 2009;338:b86. doi: 10.1136/bmj.b86.

11 Jørgensen KJ, Gøtzsche PC. Presentation on websites of possible benefits and harms from screening for breast cancer: cross sectional study. BMJ. 2004;328(7432):148.

12 Heath I. Life and Death. It is not wrong to say no. BMJ. 2009;338:b2529. doi: 10.1136/bmj.b2529.

13 Gigerenzer G, Mata J, Frank R. Public knowledge of benefits of breast and prostate cancer screening in Europe. J Natl Cancer Inst. 2009;101(17):1216-20.

14 Domenighetti G, D’Avanzo B, Egger M, et al. Women's perception of the benefits of mammography screening: population-based survey in four countries. Int J Epidemiol. 2003;32:816-21.

15 Wegwarth O, Gigerenzer G. Less is more: Overdiagnosis and overtreatment: evaluation of what physicians tell their patients about screening harms. JAMA. Intern Med. 2013;173(22):2086-7.

16 Wegwarth O, Schwartz LM, Woloshin S, Gaissmaier W, Gigerenzer G. Do physicians understand cancer screening statistics? A national survey of primary care physicians in the United States. Ann Intern Med. 2012;156(5):340-9.

17 Roberts M. Breast screening: time for a rethink? BMJ. 1989;299:1153-5. 